\title{
Alfabetización económica y financiera en el contexto escolar: Perspectivas y propuestas
}

\author{
Susana FERNÁNDEZ-LARRAGUETA, Juan ALMAGRO-LOMINCHAR \\ y Juan FERNÁNDEZ SIERRA
}

\author{
Universidad de Almería
}

(Recibido el 21 de Mayo de 2018; Aceptado el 25 de Julio de 2018)

RESUMEN: En la actualidad, la dimensión económico-financiera ha tomado fuerza en el proceso de recomposición curricular y de formación del profesorado, principalmente, debido al impulso de organizaciones económicas, cuyos informes incorporan, cada vez en mayor medida, los criterios de los que han de partir las políticas educativas que abordan estos contenidos en el escenario escolar. En este sentido, este estudio presenta parte de los resultados de una investigación realizada en dos centros andaluces de Educación Secundaria y Bachillerato, entre cuyos objetivos destacamos dos: Indagar el reflejo de las directrices de organismos económicos en los planes educativos de Economía y estudiar la incidencia de las mismas en la formación del profesorado que imparte estas asignaturas. La investigación se plantea desde un enfoque metodológico cualitativo-interpretativo, a partir de lo que Stake (2007) denomina estudio de caso instrumental. Para abordar la investigación, desde esta perspectiva, hemos utilizado, como principales técnicas para la recogida de información, entrevistas semiestructuradas, observación participante y análisis de documentos.

Los resultados obtenidos evidencian que organismos económicos, como la OCDE, elaboran en sus informes directrices cuyo reflejo es evidente en el devenir curricular de las asignaturas económicas, así como en la formación del profesorado que imparte y trabaja estas materias en el contexto escolar.

Palabras clave: Educación financiera, organismos económicos, currículum escolar, formación del profesorado.

\section{Economic and financial literacy in the school context: Perspectives and proposals}

\begin{abstract}
At present, the economic-financial dimension has taken on the process of curricular recomposition and teacher training, mainly due to the impulse of economic organizations, whose reports incorporate, increasingly, the criteria of those who have to leave the educational policies that address these contents on the school stage. In this sense, this study presents part of the results of a research carried out in two Andalusian centres of centres of secondary and baccalaureate education, among whose objectives we emphasize two: to investigate the reflection of the guidelines of economic organisms in the educational plans of economy and to study the incidence of the same in the training of teachers who teach these subjects. The research is approached from a methodological qualitative-
\end{abstract}


interpretative approach, starting from what Stake (2007) calls instrumental case study. To address research, from this perspective, we have used, as the main techniques for information gathering, semistructured interviews, participant observation and document analysis.

The results obtained show that economic organizations, such as the OECD, elaborate in their reports guidelines whose reflection is evident in the future curricular of the economic subjects, as well as in the training of the teachers who teaches and works these matters in the school context.

Keywords: Financial education, Economic agencies, school curriculum, teacher training

Correspondencia: Juan Almagro-Lominchar. jal232@ual.es

\section{Introducción}

Estudios como los de Navarro (2015), Polanyi (2009) o Herrera Flores (2001), coinciden en que la economía que rige el funcionamiento del mundo económico, político y social a ambos lados del Atlántico Norte -EEUU. y Europa Occidental- presenta una evidente tendencia neoliberal. Desde esta perspectiva, la economía pasa de ser una ciencia que posibilita el estudio de las necesidades humanas, a partir de los conceptos de democracia y justicia social (Riádigos Mosquera, 2015), a un espacio, absolutamente diferenciado de lo social, regido por las corrientes financieras, en el que cada sujeto ve reducida su actividad a satisfacer sus propias necesidades. Como sostiene, en este sentido, Herrera Flores (2000, p.1), "el individuo egoísta como sujeto; y la ganancia como norma de actuación". Entendidos desde esta perspectiva, los asuntos económicos imposibilitan la puesta en escena de mecanismos que faciliten su comprensión desde una visión holística, de la cual emerja un marcado cariz colectivo y, fundamentalmente, ético-cívico, que vaya más allá de las necesidades y deseos individuales (Navarro, 2015). En otras palabras: si concebimos la economía -más allá de asociarla a estrategias de producción y consumo- como una ciencia social que ha de satisfacer las necesidades humanas, comprendidas desde ese rigor ético y democrático que describe Sampedro (2009), resulta imprescindible trabajar y enfocar los asuntos económicos a partir de parámetros que humanicen dicha ciencia, pues de lo contrario, concluye Polanyi (2009, p.102), "cualquier discusión sobre la naturaleza de los deseos y necesidades humanas en general carece completamente de sentido".

Las consecuencias de los asuntos y problemas económicos, han ido adquiriendo un significativo protagonismo en nuestra realidad cotidiana, a pesar de esa perspectiva reduccionista que los convierte en un compartimento abstracto e independiente. Un hecho que viene a justificar, por parte de la legislación educativa, la incorporación de nociones económicas desde las que comprender la complejidad de una ciencia social que, cada vez en mayor medida repercute en nuestra manera de pensar, relacionarnos y actuar en el mundo actual (Santisteban Fernández, 2008). En este sentido, el proceso que contempla la incorporación de contenidos específicos sobre economía al currículum educativo de Educación Secundaria y Bachillerato, presenta una dilatada trayectoria, que, a raíz de la concreción de dos asignaturas específicas de Economía (LOGSE, 1990), acaba por desligar los conocimientos económicos de otras Ciencias Humanas y Sociales, a las que habían estado vinculados tradicionalmente (Travé González, 2001), y de las cuales emerge un marcado cariz ético-cívico, necesario para comprender la realidad económica sin desvincularla del factor sociopolítico que la determina (Navarro yTorres, 2012).

(c) Psy, Soc, \& Educ, 2019, Vol. 11(1) 
En la actualidad, este proceso de incorporación de contenidos económicos en los currícula educativos, al que venimos haciendo referencia, contempla desde hace una década, una significativa presencia en el escenario escolar de asuntos financieros, que, como trataremos de describir, llegan de la mano de organismos económicos cuyos postulados se desmarcan de la línea humanista (Sampedro, 2009), enfocando la economía desde una perspectiva neoliberalcapitalista (Hirtt, 2003; Navarro, 2015).

Somos conscientes, teniendo en cuenta los diversos estudios que se han hecho eco de este proceso de transición de contenidos económicos a financieros (Torres Santomé, 2017; Navarro, 2015; Monarca, Ball, Asprella y otros, 2015), del reflejo de la hegemonía de los postulados económico-neoliberales, en las últimas décadas, en el diseño y ejecución de los currícula educativos. Esta incidencia en los planes de estudio por parte del sistema económicofinanciero actual, ha derivado en una subordinación, tácita y explícita, de los sistemas de enseñanza a los sistemas económico-financieros (Riádigos Mosquera, 2015). Como ejemplo más significativo al respecto, nuestro trabajo trata de evidenciar el reciente y progresivo ahínco de la $\operatorname{OCDE}(2005,2012 \mathrm{~b}, 2013,2016)$ en la evaluación de la competencia financiera de los jóvenes (PISA, 2012, 2015) y la gran importancia que, en el caso del Estado español, el Ministerio de Educación Cultura y Deporte, junto con otras entidades de tipo económicofinanciero (Banco España, Comisión Nacional del Mercado de Valores, Asociación Española de Banca, etc.) conceden a ello, implicándose, de manera directa y explícita, en la elaboración de informes y documentos para que la educación económico-financiera tenga cada vez más visibilidad en los centros escolares.

\section{Metodología}

El artículo emerge de dos estudios de caso instrumentales, entendidos, desde los postulados de Yin $(2009$, p.18) como "un fenómeno contemporáneo dentro de su contexto real, cuando las fronteras entre el fenómeno y el contexto no son evidentes, y donde se utilizan múltiples fuentes de evidencia". El propósito de esta investigación reside en indagar desde qué postulados emerge el modelo de economía que se está enseñando actualmente en el contexto escolar de ESO y Bachillerato, así como la incidencia del mismo en la formación y labor del profesorado que trabaja estas asignaturas y en la influencia de estos contenidos en la construcción del pensamiento económico de las/os estudiantes.

De esta manera, nos hemos acercado a la realidad objeto de estudio desde un paradigma naturalista e interpretativo, ya que entendemos que la realidad social es holística y dinámica, y no es nuestro propósito establecer generalizaciones (Guba y Lincoln, 1994), sino comprender e interpretar las relaciones y los fenómenos que se establecen en dicha realidad, de acuerdo a los significados que establecen y comparten las personas que están implicadas en ella.

El estudio se ha llevado a cabo en sendos centros de Educación Secundaria y Bachillerato de la Comunidad Andaluza, de corte público y privado, respectivamente. Los instrumentos para la recogida de información se han basado en el uso de entrevistas semiestructuradas, observaciones participantes y análisis de documentos curriculares y extracurriculares. En este sentido, sin obviar la evidente relevancia de los demás instrumentos, pues suponen una herramienta fundamental para abordar la investigación desde una visión holística, hemos hecho del análisis de documentos el instrumento más relevante, debido a la 
propia naturaleza del artículo, pues este, principalmente, se fundamenta en la revisión bibliográfica de textos y otras herramientas didácticas, que, por un lado, contemplan la incorporación de las enseñanzas económicas y financieras al currículum educativo del Estado español, mientras que, por otra parte, inciden en el proceso de formación del profesorado encargado de trabajar estos contenidos económico-financieros en el espacio escolar. En aras de discutir estas cuestiones, en el artículo hemos incorporado diferentes análisis pedagógicos al respecto, elaborados por personas estrechamente vinculadas a la investigación en el ámbito educativo y de otras disciplinas del campo de las Ciencias Humanas y Sociales.

Durante este proceso de análisis de documentos y otras herramientas didácticas, hemos seguido las directrices que subyacen de estudios como los de Fernández Sierra y FernándezLarragueta (2013) y Rapley (2014). De esta manera, en concordancia con los objetivos y metodología explicados, la codificación y organización de las informaciones recogidas han dado lugar a una serie de categorías que tomamos como base para la estructuración y exposición que aquí hacemos, a saber: a) la evolución de los contenidos económico-financieros que contempla la legislación educativa, b) la incidencia de organismos económicos en el proceso que reconoce la Educación Financiera como parte del programa educativo de las asignaturas económicas, c) el enfoque desde el cual emerge la formación del profesorado que ha de trabajar estas materias económicas y d) el planteamiento de alternativas desde las que abordar el conocimiento económico-financiero en el escenario escolar.

\section{Resultados y discusión}

Las siguientes líneas describen las evidencias del estudio, elaboradas a partir de las categorías definidas anteriormente, y a las que hemos incorporado elementos teóricos con un potencial significativamente explicativo.

\section{a) La trayectoria de la Economía como disciplina escolar: Una revisión de los contenidos económicos a través de la legislación educativa}

Estudios como los de Travé González $(1999,2001)$ indagan cómo, con anterioridad a la Ley Orgánica General del Sistema Educativo (LOGSE), de 1990, los contenidos de economía estaban vinculados a disciplinas de Derecho y a otras Ciencias Sociales, como Historia y Geografía, principalmente, a raíz de que fuesen sucediéndose los diferentes acontecimientos de los que emergen periodos históricos de emancipación social.

Si nos remontamos a 1857, cuando se aprueba la Ley Moyano, encontramos que las nociones sobre economía estaban vinculadas a materias como el Derecho Político Penal y Procesal, Estadística Comercial, Agronomía y Nociones Generales de las Industrias o Agricultura y Técnicas Agrícolas. Así lo sustentan estudios como los de Capel y Urteaga (1986). Fue con posterioridad, con la II República, a partir de 1931, cuando la legislación educativa vincula las nociones económicas, en mayor medida, a la Historia Política, orientando los planes educativos hacia una enseñanza de la Historia Universal y de España, que contemplaba aspectos sociales, pero también económicos, relacionados con las características del régimen capitalista y el surgimiento de una clase obrera organizada política, social y económicamente en el contexto de la edad contemporánea (Travé González, 2001). 
Posteriormente, en los albores de la Guerra Civil (1936-1939) se vislumbran algunas de las modificaciones en los planes de estudios que el gobierno republicano lleva a cabo en pleno conflicto, cuando la enseñanza de la economía se integra, junto a Geografía e Historia, en un bloque de contenidos orientados al desarrollo de valores humanos. Así, la realidad económica permanecía vinculada al factor social, de manera que las relaciones de intercambio de productos entre las diferentes fuerzas sociales y la organización de los estratos laborales recibían un enfoque orientado a conocer las causas y consecuencias de estos procesos. Finalizado el conflicto bélico, las dos disciplinas que venían aglutinando los contenidos sobre economía -Geografía e Historia-, se ciñen, por un lado, a una legislación educativa que incide en el desarrollo del Espíritu Nacional (Valero Moya, 2016), y por otro, a una enseñanza de la Economía Doméstica, elaborada por la conocida Sección Femenina, cuyos contenidos, de los que subyace un significativo carácter sexista, justifican una idea de economía vinculada al ahorro (Travé González, 1999), teniendo en cuenta el contexto social en España durante la posguerra, muy marcado por la escasa cobertura de necesidades básicas entre buena parte de la población.

Con la Ley General de Educación (LGE), de 1970, surge una significativa transformación en los planes de estudio. Gran parte de los contenidos del Bachillerato anterior pasan a integrarse en la etapa conocida como Educación General Básica (EGB). Posteriormente, surgen dos ramas de escolarización: el nuevo Bachillerato Unificado y Polivalente (BUP) y la Formación Profesional (FP). En la temática que abordamos, los contenidos sobre economía adquieren mayor presencia en el Área de Ciencias Sociales, nuevamente, de la mano de disciplinas como Historia y Geografía, a las que se unen otras materias como Filosofía. El momento histórico por el que atraviesa la economía española, principalmente debido al desarrollismo de los años posteriores a la dictadura, provoca que el Sistema Educativo en España requiera de docentes con una orientación pedagógica progresista en la enseñanza de las Ciencias Sociales (Travé González, 2001), cuyo marco teórico incorporaba, cada vez en mayor medida, contenidos de economía vinculados al conjunto de transformaciones que se estaban llevando en el modelo económico español, en aras de adaptarlo a las políticas de la Comunidad Económica Europea (CEE), que emerge del Tratado de Roma de 1957 (Sampedro, 2009).

A pesar de estos antecedentes, de los que se desprende un progresivo aumento de la presencia de contenidos económicos en el marco educativo, no fue hasta la consolidación de la LOGSE (Real Decreto 1179/1992 de 2 de octubre, BOE n²53), cuando se contempló, por primera vez, la presencia de dos asignaturas específicas de Economía en el primer y segundo curso del nuevo Bachillerato (Economía y Economía de la empresa). Un hecho que se ha ido manteniendo, e incluso incrementándose, con las sucesivas reformas educativas acontecidas en los años posteriores: Ley Orgánica de Calidad (LOCE), de 2002 y Ley Orgánica de Educación (LOE), de 2006.

Actualmente, con Ley Orgánica para la Mejora de la Calidad Educativa (LOMCE), de 2013, la enseñanza de nociones económicas incide con más fuerza en aspectos de índole empresarial y, fundamentalmente, financiera, en aras de que los contenidos sobre economía se ajusten a los requerimientos y directrices que marcan organismos económicos, como la Organización para la Cooperación y el Desarrollo Económico (OCDE). Un asunto que analizamos y desarrollamos a continuación. 


\section{b) El reflejo de las directrices de la OCDE en la evolución curricular de los contenidos económicos: De Economía a Educación Financiera}

Tal y como reflejábamos anteriormente, en la última década, los contenidos de economía han ido afianzándose hasta tal punto que, la LOMCE (2013), reconoce su presencia, no sólo en primer y segundo curso de Bachillerato, sino también en Educación Secundaria Obligatoria (ESO), concretamente en cuarto curso. Este tipo de contenidos emergen de las directrices que establece la OCDE, cuyos informes referidos a la última década, han recogido la necesidad de que la Educación Financiera tenga una mayor presencia en el contexto escolar (OCDE, 2005). En este sentido, el Ministerio de Educación Cultura y Deporte (MECD), junto a organismos económicos como la Comisión Nacional del Mercado de Valores (CNMV) y el Banco de España, además de un conjunto de entidades financieras, lideradas por la Asociación Española de Banca (AEB), la Confederación Española de Cajas de Ahorro (CECA), la Unión Nacional de Cooperativas de Crédito (UNACC), y aseguradoras privadas, como la Fundación MAPFRE, participaron conjuntamente en la elaboración de un documento conocido como Plan de Educación Financiera (en adelante, PEF), en cuyo primer periodo (2008-2012), aún bajo la vigencia de la LOE (2006), se recogen las bases para que la Educación Financiera vaya adquiriendo mayor presencia en el escenario escolar (PEF, 2008-2012).

Paralelamente, la OCDE, principal organización económica que sustenta el diseño y ejecución del PEF, con el objetivo de justificar la relevancia de incorporar la Educación Financiera a los centros escolares, venía trabajando en diferentes estudios para indagar una posible relación entre las causas de la crisis económica y la competencia financiera de los ciudadanos (OCDE, 2005). Este hecho dio lugar a una significativa novedad en el Informe del Programa Internacional para la Evaluación de Estudiantes (PISA), en las pruebas de 2012 y 2015, en las cuales se incorporó, por primera vez, un conjunto de cuestiones destinadas a medir la competencia financiera de las/os estudiantes.

En el caso del Estado español, lejos de ofrecer una respuesta satisfactoria, desde la óptica de la OCDE, los resultados en sendas pruebas dejaron constancia del déficit en competencia financiera de las/os estudiantes (OCDE, 2013, 2016; MECD, 2017). Una situación que coincide, y a la vez justifica, la renovación del PEF en su segundo periodo (2013-2017), en el cual se especifican cuatro bloques de contenidos financieros, "que han formado parte del examen PISA" (PEF, 2013-2017, p.19), ajustándose a los parámetros de estas pruebas externas, dependientes de la OCDE (ver cuadros 1-4). Dichos contenidos, en este caso, fueron los siguientes: Dinero y transacciones, Planificación y Gestión financiera, Riesgos derivados de la inversión y Perspectivas financieras (PEF, 2013-2017). 
Cuadros 1-4: Preguntas tipo. Educación Financiera. Informe PISA (2012; 2015)

DINERO PARA VIAJAR
Natalia trabaja en un restaurante 3 tardes a la
semana. Cada tarde, trabaja 4 horas y gana 10
zeds por hora. Cada semana, Natalia gana
además 80 zeds en propinas.
Natalia ahorra exactamente la mitad de la
cantidad de dinero que gana cada semana.
Natalia quiere ahorrar 600 zeds para ir de
vacaciones.
¿Cuántas semanas tardará Natalia en ahorrar 600
zeds?

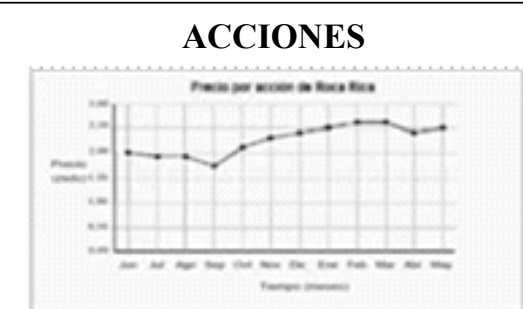

Este gráfico muestra el precio de una acción de Roca Rica a lo largo de un periodo de 12 meses

\begin{tabular}{|l|l|}
\hline \multicolumn{1}{|c|}{ Afirmación } & \multicolumn{1}{|c|}{$\begin{array}{c}\text { ¿La afirmación es } \\
\text { verdadera o falsa? }\end{array}$} \\
\hline $\begin{array}{l}\text { El mejor mes para comprar las } \\
\text { acciones fue septiembre. }\end{array}$ & Verdadero / Falso \\
\hline $\begin{array}{l}\text { El precio de la acción aumento } \\
\text { aproximadamente un 50\% a lo } \\
\text { largo del año. }\end{array}$ & Verdadero / Falso \\
\hline
\end{tabular}

OPCIONES DE GASTO
Clara y sus amigas van a alquilar una casa.
Todas llevan dos meses trabajando. No tienen
ahorros. A todas les pagan mensualmente y
acaban de recibir sus sueldos. Han preparado
esta lista de "Asuntos pendientes":
• Contratar TV por cable
• Pagar el alquiler
• Comprar muebles de exterior
¿Qué tarea o tareas de la lista es probable que
requieran atención prioritaria de Clara y sus
amigas?

\section{NUEVA OFERTA}

La Sra. Jiene un préstamo de 8.000 zeds de la Financiera Primazed. La tasa de interés anual del préstamo es del $15 \%$. Los pagos mensuales son de 150 zeds. Pasado un año, la Sra. Janeiro todavía debe 7.400 zeds. Otra empresa financiera, llamada Zedsúper, le ofrece a la Sra. Janeiro un préstamo de 10.000 zeds con una tasa de interés anual del $13 \%$. Los pagos mensuales también serían de 150 zeds.

¿Qué posible desventaja financiera puede tener para la Sra. Janeiro aceptar el crédito de Zedsúper?

Fuente: OCDE (2012b); OCDE (2016)

Es también durante este periodo (2013-2017), coincidiendo con la consolidación de la LOMCE (2013), cuando la Educación Financiera que emerge de las directrices de estos organismos económicos (OCDE, CNMV y Banco de España) e instituciones y entidades financieras (AEB, CECA, UNACC, Fundación MAPFRE), comienza a tener una mayor presencia en el currículum educativo, dentro de la asignatura de Economía (MECD, 2017), y por consiguiente, un reflejo cada vez más significativo en el escenario escolar. Así lo evidencian algunos de los materiales didácticos de Educación Secundaria y Bachillerato que se analizaron durante el estudio, en los que, por ejemplo, coincidiendo con los textos que recoge el PEF (2013-2017), cuando refleja que "la baja tasa de natalidad y el aumento de la esperanza de vida, unidas a la época de crisis, hacen necesario concienciar a los individuos para que complementen la pensión pública que recibirán una vez jubilados” (p.20), se hace 
referencia explícita a "la necesidad de contratar un plan de pensiones privado cuanto antes" (Extr.ana.doc.) ${ }^{1}$.

Es así como, recapitulando la información que contiene nuestro análisis, somos conscientes de que la Educación Financiera que establece la OCDE a través del PEF, está, cada vez en mayor medida, imbricándose en el Marco Educativo de las asignaturas de Economía que la LOMCE (2013) reconoce en ESO y Bachillerato, y por ende, en el contexto escolar. Una evidencia que, actualmente, vuelve a tomar relevancia, pues mientras redactamos estas líneas, hemos sido conocedores de una nueva renovación del PEF en su tercer periodo (2018-2021) (BOE, núm.36 de 9 de febrero de 2018), lo cual nos lleva a pensar que este proceso continuará durante los próximos años, quizá incluso, si atendemos a las palabras de Domínguez Martínez (2013) en ciclos anteriores a ESO y Bachillerato, ya que "La educación financiera se concibe como un proceso continuo, a ser desarrollado a lo largo de la vida, y que conviene comenzar a una edad lo más temprana posible, preferentemente dentro del sistema educativo" (p.73).

\section{c) La formación del profesorado que imparte las asignaturas económicas en ESO y Bachillerato: ¿De la mano de quién?}

De la misma manera que, tal y como venimos apuntando, resulta evidente el reflejo de las directrices de la OCDE, la CNMV y el Banco de España en el proceso de transición de la alfabetización económica a la alfabetización financiera en el currículum educativo, bajo la óptica de estas instituciones también se han elaborado una serie de módulos para la formación del profesorado encargado de impartir los contenidos y asuntos económico-financieros en el contexto escolar. Estos módulos, a los cuales se puede acceder a través de las webs finanzasparatodos.es y gepeese.es, incorporan un Programa de Educación Financiera (20172018) dotado de recursos y contenidos con la finalidad de contribuir a facilitar la labor pedagógica del profesorado y al enriquecimiento de sus clases (PEF, 2008-2012, 2013-2017, 2018-2021).

Los contenidos para la formación financiera de las y los docentes del Área de Economía que este denominado Programa de Educación Financiera (2017-2018) recoge, inciden en la motivación de las/os estudiantes, por parte del profesorado, en asuntos como: la gestión de sus finanzas; el fomento de buenos hábitos de ahorro durante su vida; el papel de las entidades bancarias y la financiación por parte de estas como herramienta para adquirir ciertos productos $\mathrm{y}$ servicios financieros, tales como préstamos hipotecarios y préstamos personales; las consecuencias y el impacto del endeudamiento entre la ciudadanía; el uso de las tarjetas de crédito como herramienta financiera alternativa al dinero físico; y las posibilidades para aumentar la renta personal mediante estrategias de inversión en depósitos de renta fija $\mathrm{o}$ variable, fondos de inversión y planes de jubilación como producto de ahorro-previsión.

En este sentido, consideramos que, a la vista de los contenidos y asuntos que conforman estos módulos, destinados a la formación del profesorado, bajo el propósito de que las y los docentes incorporen la Educación Financiera en sus clases, además del evidente vínculo con los organismos económicos mencionados anteriormente (OCDE, CNMV y Banco de España), existe una conexión entre dichos módulos y algunas de las instituciones financieras que

\footnotetext{
1 La codificación (Extr.ana.doc), referida al análisis de un documento (libro de texto de Economía) con el que las y los estudiantes de uno de los estudios de caso trabajan en el contexto escolar.

(C) Psy, Soc, \& Educ, 2019, Vol. 11(1)
} 
colaboran en el diseño del PEF (2008-2012, 2013-2017, 2018-2021), como la Asociación Española de Banca (AEB), la Confederación Española de Cajas de Ahorro (CECA) y la Unión Nacional de Cooperativas de Crédito (UNACC), a las que ya hemos hecho alusión anteriormente. Estas instituciones agrupan a entidades financieras como el Banco Santander, Caixabank, BBVA, o Bankia, entre otras. No podemos perder de vista, en este sentido, que estas entidades financieras han constituido una de las más arduas polémicas en el reciente periodo de recesión económica, debido a una serie de prácticas carentes de ética (Sampedro, 2009; Estefanía, 2015), que han influido significativamente en el devenir de los acontecimientos sociopolíticos y económico-financieros de la última década (Verdú, 2009; Navarro y Torres, 2012; Navarro, 2015). Cuanto menos, este hecho provoca que, nuevamente en este caso, pongamos en cuestión si la formación de las y los docentes en materia de finanzas, debe ser competencia del conjunto de organismos económicos (OCDE, CNMV, Banco de España) e instituciones financieras (AEB, CECA, UNACC) que diseñan el PEF (2008-2012, 2013-2017, 2018-2021), dejando que dicha formación se produzca únicamente desde la óptica de sus directrices, o si, por el contrario, resulta necesario que este tipo de programas para la formación financiera de las y los docentes sean contrastados desde otros discursos, enraizados en los análisis pedagógicos que consideran y conciben las políticas educativas, en todos sus ámbitos y niveles, como una herramienta para la transformación y mejora de la realidad social (Torres Santomé, 2001, 2017; Riádigos Mosquera, 2015; Fernández Sierra, 2011).

\section{d) Otra Economía es posible: Algunas propuestas desde una perspectiva pedagógica de justicia social y democracia}

A pesar de este significativo incremento en lo referido a cuestiones económicofinancieras en la trayectoria educativa del marco curricular, las cuales, como hemos evidenciado, llegan de la mano de organismos económicos e instituciones financieras con unos intereses definidos y cosificados para reproducir el modelo económico actual (Torres Santomé, 2017), resulta necesario que consideremos los asuntos y contenidos económicos, a partir de una perspectiva pedagógica que abogue por establecer políticas educativas desde las que aproximarnos a alcanzar la justicia social que requiere una sociedad formalmente democrática (Fernández Sierra, 2011; Riádigos Mosquera, 2015).

En este sentido, desde una perspectiva pedagógica que concibe la Economía como una disciplina escolar para la comprensión de los fenómenos económicos y los problemas que de éstos derivan (Travé González, 2001), no podemos obviar que esos fenómenos suceden en un escenario social diverso, en el que tienen lugar una serie de relaciones entre personas, grupos y sociedades. Como sostiene Pagès (2005, p.45):

Hoy no es posible entender el mundo ni poder participar en su transformación sin aquellos conocimientos económicos, y también de otras disciplinas sociales, que expliquen a los jóvenes y a la ciudadanía en general: a) las causas por las que la ciudadanía ha renunciado a su poder transformador limitándose a producir y consumir y, de vez en cuando, a votar; las desigualdades sociales y económicas, la marginalización y la exclusión, el paro y la pobreza; c) las migraciones masivas y d) la injusticia del sistema económico mundial con el aumento de la 
miseria de gran parte de la humanidad y la creciente violencia como respuesta a cualquier tipo de problemas.

Así, si la Economía como asignatura absorbe, sin más, las directrices de organismos económicos como la OCDE, y se reduce a plantear contenidos como el dinero y las transacciones, las nociones de inversión, la gestión financiera o los productos y servicios financieros necesarios para completar la pensión pública (PEF 2013-2017), entre otros, tratando de manera superflua la desigualdad subyacente de un sistema económico financiero como el actual, bajo los parámetros del neoliberalismo (Navarro, 2015) y, en muchos casos, obviando conocimientos que emergen de problemas socialmente relevantes, como los que expone Páges (2005), en lugar de ayudar a comprender la complejidad de los asuntos económicos y facilitar su transformación, con el propósito de contribuir a erradicar ciertas desigualdades sociales inherentes al modelo económico actual, se favorece la ocultación de dicha complejidad y la economía entra en nuestras aulas, no sólo de manera abstracta, favoreciendo la legitimación del statu quo de dicho sistema, sino también aséptica, inhibiendo una posible actuación por parte de las/os estudiantes en el proceso de comprensión y transformación social.

En consideración con este tipo de reflexiones, hemos de tener en cuenta que, mientras la legislación educativa defiende la incorporación de conceptos micro y macroeconómicos bajo la disposición que presentan las asignaturas del área de Economía, el último Foro de Davos (2018) ha vuelto a advertirnos del incremento de los niveles de pobreza en el planeta; de que allí donde las cifras de paro no continúan disparándose, sí lo hace la precariedad del empleo y de que el medio ambiente no ceja en su empeño de emitir señales de riesgo con las que alertarnos ante un futuro, cuanto menos incierto (Taibo, 2011; Riádigos Mosquera, 2015). Para explicar y profundizar en cuestiones de este calado, es imprescindible la interrelación de la Economía con otras disciplinas del campo de las Ciencias Sociales como la Filosofía, desde la que llegar a la reflexión y a la búsqueda del análisis crítico de la realidad económica; o la Sociología, a partir de la que explicar cómo la economía colaborativa está adquiriendo cada vez más relevancia en las relaciones sociales y en los comportamientos de las personas, apostando por el intercambio de bienes y servicios en lugar del tradicional concepto de propiedad. Pero también es importante visualizar la Economía en relación con las Ciencias Naturales, dado que salvaguardar los recursos del Planeta, apostando, por ejemplo, por el uso de energías renovables, es fundamental para el desarrollo de nuestras generaciones venideras (Taibo, 2011).

Desde esta perspectiva, consideramos, en aras de alcanzar un modelo educativo diferente, problematizar los asuntos económico-financieros que entran nuestras aulas, a partir de una revisión de la actual estructura pedagógica curricular que asocia estos contenidos de Economía, cada vez en mayor medida, a asuntos referidos a la Educación Financiera, únicamente desde la óptica de la OCDE y otras instituciones del ámbito económico y de las finanzas, desde la cual, resulta complejo abordar problemáticas socialmente relevantes como las que, entre otras, expone el Foro de Davos (2018). Como sostiene Fernández Sierra (2011, p.72), "Es preciso un cambio en la organización del conocimiento de los currícula educativos, representándolos en torno a cuestiones o temas candentes y relevantes de la sociedad actual".

El contexto sociopolítico actual facilita la posibilidad de que, del mundo económicofinanciero, emerjan esas cuestiones o temas candentes a los que se refiere Fernández Sierra (2011), a partir de los cuales, la legislación educativa, más allá de incrementar los contenidos 
de las disciplinas del ámbito de la economía y las finanzas, considere la posibilidad de profundizar en aquellos asuntos relevantes, vinculados a la ética, la democracia y la justicia social, que permitan al profesorado del área de Economía de Educación Secundaria y Bachillerato, enfocar y trabajar los contenidos económico-financieros desde una perspectiva ético-cívica, crítica e interdisciplinar.

\section{Conclusiones}

Las líneas que recoge este texto indagan la evolución de los contenidos económicos que la legislación (Ley Moyano, 1857;LGE, 1970; LOGSE, 1990; LOCE, 2002; LOE, 2006; LOMCE, 2013) ha ido incorporando al currículum educativo y al escenario escolar. Estos contenidos, que permanecían adheridos a otras Ciencias Sociales, como Geografía e Historia durante la Ley Moyano (1857) y la LGE (1970), quedaron desvinculados de estas con la LOGSE (1990), cuando la Economía se convierte, por primera vez, en una asignatura independiente (Travé González, 2001). A partir de ese momento, los contenidos económicos, no sólo se consolidan dentro del Marco Legislativo que regula la educación, sino que, con las leyes posteriores (LOCE, 2002; LOE, 2006; LOMCE, 2013), comienzan a sentarse las bases de un significativo proceso en el que las disciplinas económicas recogen, cada vez en mayor medida, contenidos referidos al mundo de la empresa (Hirtt, 2003), y, principalmente, de las finanzas. Es así como, desde el año 2008, con la LOE (2006), se pone en marcha el primer periodo del PEF (2008-2012), que con el aval de la OCDE, la CNMV y el Banco de España, pone sobre la mesa una serie de directrices diseñadas por instituciones financieras y aseguradoras privadas, como la Asociación Española de Banca (AEB) y la Fundación MAPFRE, con el objetivo de que la Educación Financiera se incorpore, "lo antes posible" (OCDE, 2005, p.10) en los centros escolares. Las evaluaciones externas de PISA $(2012,2015)$, en las que se establece un programa piloto que mide la competencia financiera de las y los estudiantes, así como la lectura que se hace de los resultados de dichas pruebas en el Estado español (OCDE, 2013, 2016; MECD, 2017), coinciden con la renovación y consolidación de una segunda parte del PEF (2013-2017), ya bajo el periodo legislativo de la LOMCE (2013). Actualmente, este documento ha renovado nuevamente sus bases (2018-2021), confirmando, como manteníamos con anterioridad, la consolidación de la Educación Financiera en el currículum educativo y en el espacio escolar, Entre los contenidos financieros que recogen estos textos, encontramos asuntos referidos al dinero, la gestión financiera, los productos y servicios financieros y las nociones de inversión (PEF, 2013-2017)

De la misma manera que estos organismos económicos (OCDE, CNMV y Banco de España) e instituciones financieras (lideradas por la AEB), han incidido, significativamente, en la transición de contenidos económicos a financieros, fuertemente afianzados tal y como refleja el currículum educativo actual (MECD, 2017), también han participado en el diseño de una serie de módulos para la formación en finanzas del profesorado encargado de impartir este tipo de contenidos en el espacio escolar. Unos módulos en los que se hace referencia a cuestiones como: la gestión de las finanzas por parte de las/os estudiantes, el papel de las entidades bancarias y la financiación por parte de estas como herramienta para adquirir ciertos productos y servicios financieros, el uso de las tarjetas de crédito como instrumento que sustituye al dinero físico, o las posibilidades para aumentar la renta personal a través de fondos de 
inversión y planes de jubilación. Unas cuestiones que, evidentemente, favorecen los intereses de las instituciones y entidades financieras que construyen este tipo de programas para la formación del profesorado (Torres Santomé, 2017).

Frente a este tipo de directrices a las que venimos haciendo referencia, emerge el espíritu social de reflexiones y análisis pedagógicos que, en el caso de la enseñanza de contenidos sobre economía y finanzas, conceden a las políticas educativas un valor que va más allá de incidir en la concreción curricular y en la construcción de esquemas mentales económicos en docentes y estudiantes, en aras de reproducir y legitimar los intereses de los organismos, instituciones y entidades financieras que diseñan y ejecutan los programas sobre educación financiera en el escenario escolar. Así, de estas reflexiones y análisis alternativos subyace una economía que Sampedro (2009) vincula al factor humanista y ético-cívico, necesario para concienciar a las y los jóvenes de su importante rol para paliar los efectos del modelo económico neoliberal-capitalista que rige el mundo actual.

\section{Referencias}

Boletín Oficial del Estado (2018). Resolución de 23 de enero de 2018, de la Comisión Nacional del Mercado de Valores, por la que se publica el Convenio de colaboración con el Banco de España, para la promoción y el desarrollo del Plan de Educación Financiera. BOE número 36, 16428-16434. Recuperado de https://www.boe.es/boe/dias/2018/02/09/

Capel, H. y Urteaga, L. (1986). La Geografía en un currículum de Ciencias Sociales. Geocrítica, 61, 5-32.

Domínguez Martínez, J.M. (2013). Educación Financiera en la escuela: las competencias según el PISA. Revista eXtoikos, 11, 73-78.

Estefanía, J. (2015). Estos años bárbaros. Barcelona: Galaxia Guttenberg.

Fernández Sierra, J. (2011). Formar para la economía del conocimiento vs educar para la sociedad del conocimiento. Málaga: Aljibe.

Fernández Sierra y Fernández-Larragueta (2013). Investigar las transiciones académicoculturales: estudios de caso, multicaso. En J. Fernández Sierra (ed.). Transitar la cultura. Niños y niñas inmigrantes en la educación obligatoria. Estudios de caso. Multicaso (15-30). Málaga: Aljibe.

Foro de Davos (2018). World Economic Forum Annual Meeting. Extraído de https://www.weforum.org/es/events/world-economic-forum-annual-meeting-2018

Guba, E. G., y Lincoln, Y. S. (1994). Competing paradigms in qualitative research. En N. K. Denzin y Y. S. Lincoln. (Eds.), Handbook of qualitative research (pp. 105-117). California: SAGE.

Herrera Flores, J. (2000). El vuelo de Anteo. Derechos Humanos y crítica de la razón liberal. Bilbao: Desclée de Brouwer.

Hirtt, N. (2003). Los nuevos amos de la escuela. El negocio de la enseñanza. Madrid: Universitaria.

Ley Orgánica 1/1990, de 3 de octubre, de Ordenación General del Sistema Educativo. (LOGSE). (BOE núm. 238, 4 de octubre de 1990).

Ley Orgánica 10/2002, de 23 de diciembre, de Calidad de la Educación (LOCE) (BOE núm. 307, 24 de diciembre de 2002). 
Ley Orgánica 2/2006, de 3 de mayo, de Educación. (BOE núm.106 de 4 de mayo de 2006).

Ley Orgánica 8/2013, de 9 de diciembre, para la Mejora de la Calidad Educativa. (BOE núm. 295, de 10 de diciembre de 2013).

Ministerio de Educación, Cultura y Deporte (2017). Programa para la Evaluación Internacional de los Alumnos. Competencia Financiera. Informe Español. Extraído de https://www.mecd.gob.es/dctm/inee/pisa2015-competencia-financiera-inf-espanol.pdf? documentId=0901e72b825a92bd

Monarca, H. (ed.). Ball, S., Asprella, G. y otros (2015). Evaluaciones externas: mecanismos para la configuración de representaciones y prácticas en educación. Madrid: Miño y Dávila.

Navarro, V. y Torres, J. (2012). Los amos del mundo. Las armas del terrorismo financiero. Barcelona: Booket.

Navarro, V. (2015). Ataque a la democracia y al bienestar. Crítica al pensamiento económico dominante. Barcelona: Anagrama.

OCDE (2005). Recommendation on Principles and Good Practices for Financial Education and Awareness. Recuperado de http://www.oecd.org/finance/financial-education/35108560.pdf

OCDE (2012b). Ítems liberados de PISA-Competencia Financiera. OECD PISA 2012.

OCDE (2013). Programme for international student assessment (PISA). Results from PISA 2012 Financial Literacy. Extraído de https://www.oecd.org/spain/PISA-2012-results-finlit-spainesp.pdf

OCDE (2016). PISA 2015, resultados clave. Extraído de https://www.oecd.org/pisa/pisa-2015results-in-focus-ESP.pdf

Pagès, J. (2005). La educación económica de la ciudadanía. Kikiriki. Cooperación educativa. 77, $45-50$.

PISA (2012). Informe de resultados. España. Principales conclusiones. Recuperado de https://www.oecd.org/pisa/keyfindings/PISA-2012-results-spain-ESP.pdf

PISA (2015). Informe de resultados clave. Recuperado de https://www.oecd.org/pisa/pisa-2015results-in-focus-ESP.pdf

Plan de Educación Financiera (2008-2012). Comisión Nacional del Mercado de Valores y Banco de España. Eurosistema. Extraído de http://www.cnmv.es/DocPortal/Publicaciones/ PlanEducacion/PlanEducacion13 17.pdf

Plan de Educación Financiera (2013-2017). Comisión Nacional del Mercado de Valores y Banco de España. Eurosistema. Extraído de http://www.cnmv.es/DocPortal/Publicaciones/ PlanEducacion/PlanEducacion13 17.pdf

Polanyi, K. (2009). El sustento del hombre. Barcelona: Mondadori.

Programa de Educación Financiera (2017-2018). 5 preguntas y respuestas clave. Comisión Nacional del Mercado de Valores y Banco de España. Eurosistema. Extraído de http://www.finanzasparatodos.es/comun/pdf_varios/5preguntas_2017-2018.pdf

Rapley, T. (2014). Los análisis de la conversación, del discurso y de documentos en investigación cualitativa. Madrid: Morata.

Real Decreto 1179/1992, de 2 de octubre, por el que se establece el currículo del Bachillerato. BOE núm. 253, de 21 de octubre de 1992.

Riádigos Mosquera, C. (2015). Justicia social y educación democrática. Madrid: Editorial Arco/Libros-La Muralla.

Sampedro, J.L. (2009). Economía humanista: Algo más que cifras. Madrid: Debolsillo. 
Santisteban Fernández, A. (2008). La educación para la ciudadanía económica: comprender para actuar. Íber: Didáctica de las Ciencias Sociales, Geografia e Historia, 13(58), 16-25.

Stake, R. (2007). Investigación con estudios de caso. Madrid: Morata.

Taibo C. (2011). El decrecimiento explicado con sencillez. Madrid: La Catarata.

Torres Santomé, J. (2001). Educación en tiempos de neoliberalimo. Madrid: Morata.

Torres Santomé, J. (2017). Políticas educativas y construcción de personalidades neoliberales y neocolonialistas. Madrid: Morata.

Travé González, G. (1999). La Economía y su didáctica en la educación obligatoria. Sevilla: Diada.

Travé Gónzalez, G. (2001). Didáctica de la economía en el bachillerato. Madrid: Síntesis.

Valero Moya, A. (2016). Los manuales de pedagogía y la formación pedagógica de los maestros durante el primer franquismo (1939-1960), Microespacios de investigación 3, 1-34.

Verdú, V. (2009). El capitalismo funeral. La crisis o la Tercera Guerra Mundial. Barcelona: Anagrama.

Yin, R. (2009). Case Study Research, Design and Methods. California: SAGE publications. 